\title{
Participación en la escuela: Una utopía no tan lejana. Una propuesta enmarcada en el sistema educativo español ${ }^{1}$
}

\author{
Participation at school: a not so distant utopia. A proposal framed in the Spanish education \\ system $^{2}$
}

\section{Participação na escola: Uma utopia não tão distante. Uma proposta enquadrada no sistema educacional espanhol ${ }^{3}$}

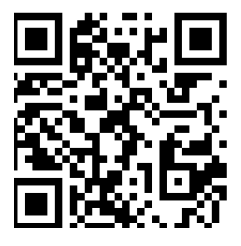

Recibido • Received • Recebido: 06 / 06 / 2018

Corregido • Revised • Revisado: 08 / 08 / 2019

Aceptado • Accepted • Aprovado: $13 / 11 / 2019$

\footnotetext{
${ }^{1}$ Documento elaborado a partir del trabajo final de grado conducente al Grado de Maestro en Educación Primaria Las claves para un aula participativa. Una propuesta para $6^{\circ}$ de educación primaria. El documento no ha sido publicado previamente ni ha sido objetivo de participaciones o ponencias (Etxebarría-Kortabarría, 2015).

${ }^{2}$ Paper elaborated from the Final Degree Project leading to the Bachelor's Degree in Primary Education The keys to a participatory classroom. A proposal for Grade 6 of Primary Education. The paper has not been previously published nor has it been the objective of participations or previous papers.

${ }^{3}$ Documento elaborado a partir do trabalho final conducente ao grau de professor do ensino fundamental. As chaves para uma sala de aula participativa. Uma proposta para a $\sigma^{a}$ série do ensino fundamental. $O$ documento não foi publicado anteriormente não era objetivo de participações ou apresentações (Etxebarría-Kortabarría, 2015).
} 
doi: http://doi.org/10.15359/ree.24-1.12

URL: http://www.una.ac.cr/educare

CORREO: educare@una.cr

Resumen: La sociedad actual valora cada vez más los procesos de decisión colectiva y requiere una ciudadanía más participativa. Sin embargo, esta ciudadanía no siempre cuenta con las herramientas formativas necesarias para que la participación sea real y efectiva, lo que hace que sea fundamental potenciar la cultura participativa desde la escuela. El documento realiza, en primer lugar, un breve estudio de los conceptos de participación y participación infantil, así como del estado de la protección legal de esta última y de los principales modelos existentes para su medición, la escalera de Roger Hart y la propuesta de Novella y Trilla. Se propone, a continuación, una secuencia de actividades para ser desarrolladas por el alumnado de $6^{\circ}$ de educación primaria del sistema educativo español durante un curso académico. Con la propuesta, flexible y adaptable a otros cursos de educación primaria o al primer ciclo de educación secundaria del mismo sistema educativo, se pretende potenciar, de un modo ordenado y sistemático, las competencias participativas del alumnado dotándolo, así, de herramientas concretas para su participación social y ciudadana presente y futura.

Palabras claves: Participación del estudiantado; instrumento de medida; derechos de la niñez; enseñanza primaria; enseñanza secundaria.

\begin{abstract}
Today's society increasingly values collective decision-making processes and requires more participatory citizens. However, these citizens do not always have the necessary training tools so that participation is real and effective, which makes it fundamental to promote the participatory culture from the school. The document first presents a brief study of the concepts of participation and child participation, as well as the status of legal protection of the latter and the main existing models for its measurement, the Roger Hart staircase, and the Novella and Trilla proposal. It is proposed, next, a sequence of activities to be developed by students of 6th grade of Primary Education in the Spanish education system during an academic year. With the proposal, flexible and adaptable to other Primary Education courses or to the first cycle of Secondary Education in the same education system, the aim is to promote, in an orderly and systematic way, the participatory competences of the students, providing them with specific tools for their present and future social and civic participation.
\end{abstract}

Keywords: Students participation; measuring instrument; rights of the child; primary education; secondary education.

Resumo: A sociedade atual valoriza cada vez mais os processos de decisão coletiva e requerem cidadãos mais participativos. Porém, esta cidadania nem sempre conta com as ferramentas formativas necessárias para que sua participação seja real e efetiva, indicando a importância de potencializar a cultura participativa desde a escola. A pesquisa realiza, primeiramente, um breve estudo dos conceitos de participação e participação infantil, como também o estado da proteção legal desta última e dos principais modelos de medição existentes, a escala de Roger Hart e a proposta de Novella e Trilla. Posteriormente, a proposta gira em torno a uma sequencia de atividades para serem desenvolvidas pelos estudantes do $6^{\circ}$ grau de educação primária do sistema educacional espanhol durante um curso acadêmico. Com uma proposta flexível e adaptável a outros cursos de ensino fundamental ou médio no mesmo sistema educativo, pretende-se potencializar, de um modo ordenado e sistemático, as competências participativas dos estudantes, ajudando assim com materiais concretos, a uma participação social e cidadã presente e futura.

Palavras chave: Participação dos estudantes; instrumento de medida; direitos da infância; ensino fundamental; ensino médio. 


\section{Introducción}

La sociedad actual reclama una mayor participación en ámbitos tan dispares como son la política, las comunidades vecinales o las asociaciones de madres y padres del alumnado. Son muchos los ejemplos que nos indican esta realidad, desde un incremento notable del uso de las redes sociales, pasando por el aumento de la participación ciudadana en las aprobaciones de presupuestos de distintos ayuntamientos, hasta llegar a movimientos sociopolíticos tan mediáticos como pueden ser el 15-M o la Primavera Árabe. Estos ejemplos demuestran que la sociedad en la que vivimos y de la que somos parte está en un proceso de continua evolución y que cada día requiere más participación en aquellos asuntos que le sean de interés o resulten cercanos.

Señala Hart (1997) que la participación es la capacidad para expresar decisiones que sean reconocidas por el entorno social y que afectan la vida propia o la vida de la comunidad en la que se vive. Y, añade Hart (1997), esta capacidad también es propia de la niñez: destaca lo mucho que los niños y las niñas participan en los temas de su interés.

Ahora bien, pese a que la participación no tiene edad, la realidad es que el interés y el ánimo por tomar parte en las decisiones colectivas no son aptitudes con las que se nace, hay que aprender a participar. En la escuela actual se han dado pasos para integrar una cultura de la participación dentro del proceso de enseñanza-aprendizaje del alumnado y es que, siguiendo la terminología de Puig (1996), la escuela es cada vez más una "comunidad participativa". Sin embargo, es necesario ir más allá, pues conviene que el alumnado se familiarice de un modo continuado y gradual con el concepto de participación y con su práctica, de modo que pueda llegar a participar plenamente no solo en la escuela, sino también, en el futuro, en un ámbito social más amplio. Conviene, por tanto, potenciar la cultura participativa desde las aulas.

Así, partiendo de la importancia que tiene fomentar (y afianzar) la cultura de la participación en los grupos más jóvenes, se ha realizado un pequeño estado de la cuestión en el que se analiza lo que se entiende por participación y los niveles de protección y medición de la participación infantil. A continuación, se ha realizado una propuesta concreta de actuación para fomentar la participación del alumnado en el marco del último curso de la educación primaria del sistema educativo español.

\section{Participación y sociedad}

La palabra participar proviene del término latino participare y su significado, siguiendo lo dispuesto por la Real Academia de la Lengua Española, RAE (2001), puede sintetizarse en tres acepciones principales. En primer lugar, tomar parte en algo, es decir, compartir con otros miembros de un grupo concreto decisiones que tienen que ver con un interés común (RAE, 2001). Participar significa también recibir una parte de algo, por ejemplo, de las ganancias de un 
doi: http://doi.org/10.15359/ree.24-1.12

URL: http://www.una.ac.cr/educare

CORREO: educare@una.cr

negocio o del éxito de una acción. Finalmente, el término implica compartir y tener las mismas opiniones e ideas que otros individuos. Podríamos decir, por lo tanto, que participar se cimienta fundamentalmente en tres ideas básicas: 1) tomar parte en algo, 2) recibir y 3) compartir. Las tres con una gran imbricación en el ámbito de lo social.

Han sido numerosas los estudios que se han acercado al tema de la participación, especialmente en las esferas política y ciudadana (Blondiaux, 2008; Cunill, 1991; Morán, 2018), y las grandes visiones de aquella; la participación como derecho político y la participación como proceso de formación de opiniones, refuerzan esa idea social del término.

En cuanto a la participación como derecho político, quizás la definición más clásica sea la de Verba y Nie (1972), quienes identificaron la participación política con aquellas actividades de la ciudadanía que está más o menos directamente destinada a influir en la elección de las personas que la gobiernan y en las acciones que adoptan. Sin embargo, hoy buena parte de las acciones de participación política no se dirigen directamente al gobierno. Como nos recuerdan Torcal, Montero y Teorell (2006), el boicot, por ejemplo, a un producto concreto con el fin de influir en los medios de producción de una determinada empresa, también ha de ser considerado como participación política, aunque la finalidad no sea influir en quienes gobiernan. La definición de Brady (1999), que pone el acento en el objetivo de influencia del proceso de participación política y no en aquellos a quienes va dirigida, parece hoy, pues, más adecuada. En todo caso, como señalan Tenjo, Ribero y Sanabria (1998), se podría decir que la participación política tiene que ver con el ejercicio de la ciudadanía de aquellos derechos que les son reconocidos constitucionalmente. Una definición amplia donde encajan los deseos de influir en las élites, sean estas cuales sean.

La participación vista como proceso de formación de opiniones es la visión clásica de autores como Mills (1954, citado en Ministerio de Educación Política Social y Deporte, Secretaría de Estado de Política Social, Familias y Atención a la Dependencia y a la Discapacidad e IMSERSO, 2008), quien apunta en su obra a la participación como el proceso de puesta en común de ideas y soluciones en torno a problemas comunes en el marco de los grupos de trabajo. Resulta interesante en este autor la idea de participación como proceso permanente que culmina con la toma de decisiones. Es decir, pasar de lo teórico a lo práctico. En la misma línea, Gyarmati (1992) focalizó la participación en aspectos que afectan al individuo en relación con el grupo del que forma parte, muy especialmente en el trabajo; pero también en otras esferas sociales. De nuevo, vemos la idea de imbricación permanente de la participación con la sociedad.

Se puede señalar, por tanto, que la participación -se vea en su vertiente política o como formadora de opinión en el ámbito colectivo- es un proceso, en el que mediante pequeños acontecimientos y hechos se busca un resultado o una meta para tratar temas de interés común. Y dado que la participación no puede ser algo individual, sino social, esta requerirá de dos elementos: 
1) que determinados sujetos actores entren en contacto, ya sea bien un pequeño grupo de gente, bien una comunidad entera; y 2) que existan actitudes de escucha y respeto de las opiniones de todas las partes, pues la participación supone tomar decisiones y gestionar recursos.

La relación entre sociedad y participación es tan intensa, que todo parece indicar que las personas, por el hecho de formar parte de la primera, estamos en un continuo proceso participativo. $Y$ es que votando, acudiendo a una manifestación o poniéndonos una chapa en la camiseta, opinamos y actuamos en relación con las cuestiones que nos preocupan y nos interesan. Ahora bien, como apunta Novella (2012), este planteamiento no es del todo real y efectivo. Hay muchas formas y niveles de participación (Blas e lbarra, 2006) y, como señalan Trilla y Novella (2001), la lectura habitual de citas como "ha habido mucha participación", "han estado muy participativos" o "hay que promover la participación" no debe de implicar gran cosa, a no ser que vengan acompañadas con datos concretos. La palabra participación, para estos estudios, en un término "prestigiado" con el que se hace mucha "demagogia y retórica vacía", lo que nos lleva al punto opuesto al que quieren llegar sus portavoces, al del hastío o desconfianza que generan algunas experiencias participativas, como nos recuerdan Jiménez y García (2015).

Para evitar esta deriva, siguiendo a Novella (2012), consideramos que debería ser una empresa colectiva buscar cómo la educación puede contribuir a la construcción y puesta en marcha de mecanismos y procesos participativos. Tomando la idea de participación como poder (Dale, 1999; Gaitán, 1998), así como el éxito de diversas experiencias previas (Castilla, 2016; de la Riva y Moreno, 2018; García y Prado, 2013), parece clara la necesidad de empoderar a la sociedad en esta cuestión, incluyendo también a las personas más pequeñas, para volver a llenar el término de significado.

\section{La participación infantil: Protección legal y modelos para su medición}

La participación infantil es, hoy en día, un derecho de la niñez y personas adolescentes que les es reconocido por diversos instrumentos legales. Y esto es así, señala Cortés (2001), desde la aprobación de la Convención de los Derechos del Niño (en adelante CDN) por la Asamblea de Naciones Unidas el 20 de noviembre de 1989. Desde este momento, comenzó a visibilizarse a la niñez como sujeto de derechos que debían ser sometidos a protección. Se les reconoció, señalan Coiduras, Balsells, Alsinet y Urrea (2016) como ciudadanos y ciudadanas. Hoy, todos los países del mundo, a excepción de Estados Unidos, han aceptado cumplir las normas de este tratado que reconoce como sujetos de derechos a los menores de 18 años. Y resulta interesante destacar que su aplicación es obligación de los gobiernos, pero también define las obligaciones y responsabilidades de otros sujetos como los padres, madres, profesorado, profesionales de la salud, grupos de investigación, así como los propios niños y niñas. 
doi: http://doi.org/10.15359/ree.24-1.12

URL: http://www.una.ac.cr/educare

CORREO: educare@una.cr

Siguiendo el propio documento de la CDN (Naciones Unidas, 2006), los 54 artículos que la componen recogen los derechos económicos, sociales, culturales, civiles y políticos de todos los niños y las niñas, y aparecen divididos en cuatro categorías básicas: derecho a la supervivencia, al desarrollo, a la protección y a la participación. En lo que a la participación se refiere, la CDN, especialmente en sus artículos 12,13 y 15, consagra el derecho de todos los niños y las niñas a participar activamente en la comunidad y en la sociedad. Así, tendrán derecho a 1) expresar su opinión y a que esta sea tenida en cuenta, 2) recibir capacitación para poder reivindicar el cumplimiento de los artículos de la CDN y 3) asumir las responsabilidades inherentes al ejercicio de los derechos. Se trata del primer documento donde queda reconocido internacionalmente el derecho de la niñez a participar en todo aquello que le afecta.

En el caso español, el artículo 118 de la Ley Orgánica de Educación(2006) (en adelante LOE), establece que "la participación es un valor básico para la formación de ciudadanos autónomos, libres, responsables y comprometidos con los principios y valores de la Constitución" (p. 70). Además, establece que "las Administraciones educativas fomentarán, en el ámbito de su competencia, el ejercicio efectivo de la participación de alumnado, profesorado, familias y personal de administración y servicios en los centros educativos" (p. 71). Este artículo no ha sido modificado por la Ley Orgánica para la mejora de la calidad educativa (2013) (en adelante LOMCE), por lo que sigue vigente. El texto pone de manifiesto la importancia de la participación para el funcionamiento de la sociedad, y para la formación de una ciudadanía autónoma, libre, responsable y comprometida, lo que coincide con la finalidad general del sistema educativo, establecida en el artículo 2.1.K de la LOE (2006, p.16). Asimismo, parece poco probable alcanzar ese objetivo sin la colaboración y participación de todas las personas que configuran la comunidad escolar, declara esencial el trabajo en equipo y que las personas deben sentirse importantes, con facultad y capacidad de participar.

Como se ha expuesto, la normativa ampara el derecho a participar de la niñez y personas adolescentes. Ahora bien, pese a que se habla y escribe mucho sobre participación, parece que la participación infantil se ha convertido en un estandarte, en una marca de calidad, en un contenido político (Novella, 2012) y, sin duda, esto no es suficiente. Para Novella (2012) la participación infantil no es una actividad extra, algo puntual, sino que debería ser una forma de estar (niños, niñas y mayores) y de construir la identidad de la sociedad en la que vivimos. En el caso de la niñez, esta busca y logra sus propios espacios de participación, donde está en permanente relación con los grupos adultos. La cuestión es que esta participación conjunta y continua debe entenderse como un proceso de aprendizaje mutuo, entre la niñez y personas adultas, entre padres, madres y niñez, entre profesorado y niñez, entre ciudadanía adulta y niñez.

Cabe destacar aquí la importancia de la persona adulta como guía en el proceso participativo. Padres, madres, profesores y profesoras deben ser conscientes de la importancia de su rol en esta relación en pro de la participación, lo que Pérez y Figueroa (2005) denominan 
con el término entes facilitadores de procesos de decisión y comunicación. Y es que ser personas escuchadas y tenidas en cuenta y tener la posibilidad de colaborar en proyectos comunes y tomar decisiones, empodera; siguiendo de nuevo la terminología de Gaitán (1998), también a los niños y las niñas, en su relación con las personas adultas.

Se trata, por tanto, de proporcionar poder a la niñez mediante la potenciación de su participación de un modo consciente y guiado por una persona adulta. Y es que la niñez ya participa, se le permita o no. A la niñez le gusta explorar, preguntar, rastrear e indagar sobre aquello que le rodea. En general, suele tener unas ganas terribles de descubrir el mundo y para ello trata de participar en todo aquello que le afecta o le importa. Así, desde bien pequeña la niñez empieza a expresar sus opiniones e inquietudes sobre las cuestiones que le afectan porque tienen que ver con su familia, o su entorno escolar o social. Es decir, poco a poco y a medida que van creciendo, de una forma algo inconsciente, los niños y las niñas suelen ir descubriendo espacios básicos donde poder participar.

Señala Hart (2001) que los espacios donde la niñez puede desarrollar su participación son fundamentalmente tres: el familiar, el escolar y el local. Pese a que los tres son importantes, por la temática de este documento, nos interesa especialmente el espacio escolar. La escuela es un lugar fundamental para potenciar la participación de sus niños, niñas y jóvenes, y es que el desarrollo de experiencias prácticas de participación infantil resulta determinante en el aprendizaje de los derechos y responsabilidades tanto individuales como colectivos.

Ahora bien, como señalan Trilla y Novella (2001), hay muchas formas, tipos, grados y niveles de participación. Así, antes de poder aplicar en el aula una propuesta didáctica dirigida a potenciar la cultura participativa de nuestro alumnado, convendrá determinar en qué nivel nos encontramos en el grupo-clase. Para medir esta cuestión resultan de gran interés la conocida como escalera de Hart (1993) o la propuesta de Trilla y Novella (2001).

En el caso de la escalera de Hart (1993), este autor se planteó cuestiones como, ¿todos los niños, las niñas y adolescentes participan de la misma forma? ¿existen distintos niveles de participación? ¿existen en nuestra sociedad espacios para una participación infantil con decisiones iniciales de las personas más pequeñas compartidas con las más adultas? Y, ¿cuál es el nivel de plena participación? Y con objeto de darles respuesta, retomó la metáfora de la escalera planteada por Arnstein (1969) y propuso su propia versión de la "escalera de la participación", en la que plantea diferentes niveles de implicación que deben ser superados para que el proceso participativo sea del todo completo, como en el caso de cualquier otro proceso adulto. Es decir, Hart (1993) planteaba utilizar una escalera con 8 peldaños para tipificar el nivel de participación de la niñez. De tal forma, con la imagen de escalera como base, Hart (1993) propone superar los tres primeros peldaños: 1) la manipulación, 2) la decoración y 3) el simbolismo, en el que la niñez no es sino un altavoz de la persona adulta y no hay una verdadera experiencia participativa, 
doi: http://doi.org/10.15359/ree.24-1.12

URL: http://www.una.ac.cr/educare

CORREO: educare@una.cr

para pasar así a los siguientes niveles de participación: 4) la participación por asignación del sujeto adulto, pero con información; 5) la participación de información y consulta (I), iniciado por las personas adultas, en las que la niñez es consultada; 6) la participación de información y consulta (II), que supone compartir decisiones con la persona menor, que hace de consejera en un diseño responsabilidad de un sujeto adulto; 6) el iniciado y dirigido por los niños y las niñas; 7) el de mayor implicación infantil, iniciado por los niños y las niñas, con decisiones compartidas con las personas adultas (Hart, 1993).

Con esta clasificación, Hart (1993) pretendía diferenciar entre la nula participación de la niñez y los procesos participativos reales, donde se tienen en cuenta las propuestas, ideas, proposiciones y planteamientos realizados por el alumnado. Como puede observarse, en los tres primeros escalones no existe una participación real. Por eso es importante plantear cuáles son los objetivos cuando se plantea llevar adelante un proceso participativo, y si uno de ellos es el que el alumnado realmente participe. Es decir, si buscamos iniciar un proceso de participación, el alumnado debe de ser un agente real en el mismo proceso, y pedirle que haga algo, a modo de espejo de nuestras propias acciones, no significa que estemos potenciando en él una cultura participativa.

Tomando a modo de referencia algunos aspectos de la escalera propuesta por Hart (1993), Trilla y Novella (2001), donde proponen una tipología diferente, partiendo de cuatro clases más amplias de participación: participación simple; participación consultiva; participación proyectiva; y metaparticipación. Es importante subrayar que se trata de cuatro maneras distintas de participar, definiéndose cada una en sus propios términos; y aunque los cuatro tipos de participación sean cualitativamente diferentes, no quiere decir que sean excluyentes entre sí. En una misma institución, actividad o proyecto pueden darse, alternativa o sucesivamente, algún tipo o varios de ellos. Asimismo, también es importante subrayar que ninguno de los cuatro tipos se puede valorar genéricamente como negativo o impropio. Tal y como afirman Trilla y Novella (2001), ninguno de los tipos de participación propuestos debe ser considerados de un modo negativo, y es que "dependiendo de múltiples factores ... un nivel teóricamente inferior de participación puede constituir el óptimo en otras circunstancias, y viceversa" (p. 144).

La participación simple es la forma más básica de participación, en la que la niñez se limita a seguir instrucciones. Así, cuando el alumnado toma apuntes, responde a preguntas o realiza actividades que simplemente exigen actuar, estamos ante una participación simple. Esta clase de participación se puede equiparar a los peldaños 3 y 4, "participación simbólica" y "asignado, pero informado", propuestos por Hart (1993) ya que en ambos casos el alumnado no participa en la realización del proyecto, aunque participe voluntariamente y sepa lo que hace. En el caso de la participación consultiva, la palabra de los sujetos es escuchada, y pasan de ser simples espectadores o ejecutantes a actores participativos, aunque todavía en un nivel básico. En este nivel se pregunta su opinión y punto de vista, esta clase de participación se puede equiparar al de los peldaños 5 y 6, "consultado e informado", propuestos por Hart (1993) ya que el profesorado no 
tiene la obligación de tener en consideración lo que dice el alumnado, ni tampoco tiene el deber de compartir las decisiones con este. La participación proyectiva, por su parte, es aquella en la que, siguiendo a Trilla y Novella (2001, p. 148) "el sujeto ... se convierte en agente". El alumnado se siente parte del proceso participativo y siente el proyecto como suyo, aunque a veces el proyecto lo comiencen unos sujetos adultos. En general, se trata de una clase de participación más exigente que las anteriores, requiere mayor compromiso y es una participación más compleja. Este tipo de participación es equiparable a los tres últimos escalones de Hart (1993). Finalmente, la metaparticipación se produce cuando los niños y las niñas consideran que sus derechos participativos no están lo suficientemente reconocidos y reclaman canales para participar. Siguiendo a Trilla y Novella (2001), se puede señalar que cuanto mayores sean la implicación, información, capacidad de decisión y responsabilidad del alumnado, más cerca estaremos de la metaparticipación (Trilla y Novella, 2001). Un ejemplo claro de la metaparticipación lo encontramos en las protestas y reivindicaciones del estudiantado en mayo de 1968 en París.

Podemos apreciar cómo, en comparación con la clasificación de Hart (1993) y su planteamiento sobre la implicación que tiene el no participar, la propuesta de Trilla y Novella (2001) se centra más en la complejidad que tiene el proceso participativo en sí. En cualquier caso, ambos planteamientos son importantes para medir, de un modo consciente, la participación de estudiantes en su día a día dentro del proceso de enseñanza-aprendizaje y es interesante que esta sea reconocida por los sujetos adultos que harán de facilitadores en su adquisición de competencias participativas.

\section{Una propuesta de actividades para potenciar la participación en $6^{\circ}$ curso de educación primaria}

Una vez realizado un breve estudio teórico sobre la participación infantil, haciendo hincapié en su protección legal y los modelos principales para llevar a cabo su medición, se presenta una propuesta didáctica. La propuesta está diseñada a modo de guía para poder ser llevada a la práctica en el aula por maestras y maestros con interés en potenciar la cultura participativa de sus estudiantes.

Mediante la propuesta didáctica se pretende que el alumnado se convierta en agente activo de su contexto escolar. Así, con la propuesta se le proporciona la potestad para expresar sus dudas y su opinión respecto a distintos temas que le afectan, con lo cual se aumenta su motivación e interés por lo que ocurre en su aula y en su centro educativo. Del mismo modo, conviene señalar que los ejercicios y actividades que se desarrollaran en clase dentro de esta propuesta didáctica han sido pensados para ser llevados a cabo por estudiantes de $6^{\circ}$ curso de educación primaria, en el marco del sistema educativo español; no obstante, la propuesta es flexible y puede ser modificada para su utilización con alumnado más pequeño o incluso del primer ciclo de educación secundaria. 
doi: http://doi.org/10.15359/ree.24-1.12

URL: http://www.una.ac.cr/educare

CORREO: educare@una.cr

\section{Objetivos}

En cuanto a los objetivos didácticos de la propuesta (ver Tabla 1)

Tabla 1: Objetivos

GENERAL ESPECÍFICOS

-Potenciar la participación activa - Interactuar en grupo mediante el diálogo.

del alumnado a través de las - Respetar las opiniones de demás compañeros y compañeras de clase.

distintas actividades propuestas - Participar en una comisión con el fin de organizar un evento.

- Cumplir de forma correcta y autónoma con aquellas tareas cuando sea responsable de ejecutarlas según calendario.

- Realizar búsqueda de información utilizando TIC de forma correcta y autónoma.

- Realizar una presentación colectiva sobre el lugar elegido para el viaje de fin de curso.

- Afianzar la autonomía personal del alumnado.

Nota: Elaboración propia.

\section{Contenidos}

Los contenidos, clasificados en los tres tipos que definió la Ley Orgánica de Ordenación General del Sistema Educativo (1990), en un intento de trabajar lo competencial más allá de lo meramente conceptual, son los que aparecen en la Tabla 2.

Tabla 2: Contenidos

\begin{tabular}{lll}
\hline CONCEPTUALES & PROCEDIMENTALES & ACTITUDINALES \\
\hline - La aportación individual al & - Capacidad de transmitir opiniones & - Colaboración y trabajo de forma \\
colectivo. & fundamentadas. & activa en el grupo \\
- El deber (y el derecho) de & - Expresión correcta y vocabulario & - Respeto al trabajo y opiniones de \\
la participación individual & adecuado (expresión escrita y oral). & las demás personas. \\
- El deber (y el derecho) de & - Organización de la información de & - Interés por las actividades que se \\
la participación grupal. & forma clara. & realizan. \\
& - Capacidad y destreza a la hora de & - Limpieza y orden en el cuaderno \\
& debatir y opinar. & y la presentación. \\
& - Aprendizaje del uso de las TIC. & \\
\hline
\end{tabular}

Nota: Elaboración propia.

\section{Temporalización y cronograma}

La propuesta está diseñada para ser puesta en práctica a lo largo de un curso escolar y se plantea, como guía, el cronograma de la Tabla 3, donde se recogen las actividades principales que se propone desarrollar con el alumnado. 


\section{Propuesta de actividades}

Se proponen a continuación cuatro actividades que pueden ser llevadas a la práctica con estudiantes durante el curso escolar. Su propósito no es otro que permitir al alumnado avanzar en las escaleras o estadios de participación propuestos por Hart (1993) y por Trilla y Novella (2001), y que se han presentado en el marco teórico. Los tiempos son indicativos.

Tabla 3: Cronograma

\begin{tabular}{|c|c|c|c|c|c|c|c|c|c|c|}
\hline Actividades/Mes & 09 & 10 & 11 & 12 & 01 & 02 & 03 & 04 & 05 & 06 \\
\hline Reglas para convivencia & $x$ & & & & & & & & & \\
\hline Elección de delegados/as & $\mathrm{X}$ & & & & & & & & & \\
\hline Buzón de sugerencias & $\mathrm{x}$ & & & & & & & & & \\
\hline Trabajar opiniones, quejas y sugerencias & & $x$ & $x$ & $x$ & $x$ & $x$ & $x$ & $x$ & $x$ & $x$ \\
\hline Definición de comisiones & $x$ & & & & & & & & & \\
\hline Reunión de comisiones & & $x$ & $x$ & $x$ & $x$ & $x$ & $x$ & $\mathrm{x}$ & $x$ & $x$ \\
\hline $\begin{array}{l}\text { Búsqueda, defensa y elección de lugar } \\
\text { para fin de curso }\end{array}$ & & & & & $x$ & $x$ & $x$ & & & \\
\hline Role playing & & & & & & & & & $x$ & \\
\hline
\end{tabular}

Nota: Elaboración propia.

Actividad 1: La primera actividad se realizará en las dos primeras clases de tutoría, en septiembre. En la primera clase de tutoría, el profesorado a cargo de la tutoría explicará en qué consiste la propuesta didáctica y qué se pretende alcanzar con ella. Es decir, los objetivos que anteriormente se han descrito, subrayando con suficiente claridad la importancia de que todo el alumnado participe en las actividades que se proponen, al igual que en el día a día de clase. (15 minutos)

Posteriormente, en grupos de 4 estudiantes se escribirán unas reglas de convivencia básicas para la clase que tendrán validez durante todo el curso escolar que acaba de comenzar. En un par de minutos el personal docente deberá orientar al alumnado, señalando la importancia que tienen conceptos como la tolerancia, el respeto y la solidaridad. (20 minutos)

Una vez que cada grupo tenga sus propias reglas de convivencia, entre todo el alumnado se redactarán las reglas básicas del aula. Un alumno o alumna, de manera voluntaria, asumirá la secretaría y escribirá en la pizarra las reglas que entre todo el grupo decidan, hasta llegar a un número de 10. A continuación, cada estudiante escribirá en su cuaderno las reglas básicas que hayan sido seleccionadas de forma unánime, y el profesor tutor o profesora tutora las recogerá en una cartulina que se expondrá en el tablón de anuncios. (25 minutos) 
doi: http://doi.org/10.15359/ree.24-1.12

URL: http://www.una.ac.cr/educare

CORREO: educare@una.cr

En la segunda sesión, dentro de esta primera actividad, se dialogará con el alumnado sobre qué entiende por participación y en qué ámbitos cree que puede participar. El profesorado les explicará la creación del buzón de opiniones, quejas y sugerencias y su funcionamiento. Es un espacio con el que se pretende familiarizar al alumnado con el concepto de la cultura participativa. (30 minutos)

A continuación, se elegirá a las dos personas delegadas de clase, mediante votación individual a mano alzada. Antes de comenzar con la votación, el personal docente a cargo explicará cuáles serán las funciones de las personas delegadas durante el curso escolar siguiente, responderá las preguntas relativas al tema y preguntará si hay estudiantes que quieran presentar su candidatura. En todo momento, el profesorado tutor tratará de asociar la votación de las personas delegadas a unas elecciones de representantes de la ciudadanía. (30 minutos)

Por último, y en cuanto al buzón de opiniones, quejas y sugerencias, semanalmente, en cada tutoría, habrá un espacio como mínimo de 10 minutos y máximo de 30 minutos para hablar de los temas que vayan surgiendo. Si alguna semana no hay aportaciones por parte del alumnado, el personal docente utilizará este espacio para hablar de temas que le resulten de interés.

Actividad 2: La última tutoría de septiembre será el día donde el alumnado tendrá que enrolarse en una comisión organizadora de eventos o de otro tipo de actividades. El profesor tutor o la profesora tutora explicará brevemente en qué consiste cada comisión y cuáles son sus deberes y funciones básicas, pudiendo ser ampliadas por aportaciones estudiantiles. Las comisiones serán de dos tipos. Unas estarán relacionadas con algún día festivo del colegio, por ejemplo: Comisión del día de la Constitución, Comisión de Navidad, Comisión de Fiesta de Carnaval, Comisión de Fiesta de Primavera, Comisión del Día del Colegio. Y otras pueden ser comisiones sueltas: Comisión del Medio Ambiente, Comisión de la Hora del Patio y Comisión Ayudemos a las personas más pequeñas. Cada estudiante elegirá en qué comisión quiere tomar parte y en caso de que exista saturación de demanda en una de las comisiones, el personal docente deberá resolver mediante votación entre los sujetos interesados. (1 hora)

Posteriormente, una vez constituidas las comisiones, cada estudiante tendrá sus reuniones respectivas en aquella de la que forme parte. Las reuniones serán semanalmente y se realizarán a la hora del recreo. En estas reuniones se encontrará con estudiantes de las otras clases del mismo curso, la idea es que las comisiones sean lo más heterogéneas posible. Una vez celebrado el evento, puede que la comisión se reúna solamente para valorar el resultado, o puede reunirse para ir perfilando otros asuntos. Lo importante es que cada comisión tome cuerpo, y que sea independiente y autosuficiente.

Actividad 3: Después de la vuelta de Navidad, el alumnado deberá elegir dónde quiere ir a pasar el viaje de fin de curso. En un principio, cualquier destino puede llegar a ser el elegido, pero para ello, deberá defender su propuesta. El viaje de fin de curso se realizará en junio, 
constará de un solo día, saldrá de su localidad al inicio de las clases y volverá a su finalización, por ejemplo, de 9:00h a17:00h. Es decir, se propone que su duración sea la de una jornada escolar. El proceso de elección del destino durará tres meses. Primero, el estudiantado deberá buscar, en grupos de 4, información en internet. Para ello se desplazarán a la sala de ordenadores en un par de clases de tutoría y por parejas, buscarán información sobre el destino previamente elegido entre 4 miembros del grupo. La información puede ser geográfica y social, pero también deberán buscar evidencias sobre actividades posibles a realizar: visita a un museo u otro lugar de interés; un paseo por el casco antiguo de la ciudad o un bosque cercano; conocer de cerca oficios antiguos, etc. Deben recoger toda la información necesaria, junto con imágenes, videos y demás aspectos que resulten de interés para la exposición grupal. (2 sesiones de 1 hora)

Una vez que tengan suficiente información del posible destino, en grupos deberán preparar una exposición, donde, mediante un PowerPoint, defenderán su candidatura. La realización de esta se realizará en la sala de ordenadores. (1 sesión de 1 hora)

Por su parte, la defensa y la presentación del destino elegido se llevará a cabo en una sesión dentro del aula. En la defensa, cada grupo expondrá las razones por las que ha elegido dicho destino, y tratará de convencer al resto para que elijan su propuesta. Al final de las 5 exposiciones se realizará una votación, en la que ningún grupo podrán votar por su propia propuesta. (1 sesión de 1 hora)

De esta forma, habrá tantos destinos posibles como clases de $6^{\circ}$ curso de educación primaria del centro educativo. A continuación, los profesores y profesoras a cargo de la tutoría se reunirán con los delegados o delegadas de curso, y entre todo el grupo, de forma unánime, elegirán un destino. Asimismo, también podrán modificar la propuesta final, siempre y cuando la propuesta inicial sea inaccesible.

Actividad 4: Consiste en un role playing. A finales de curso, en las tres semanas centrales de mayo y en clase de tutoría, el alumnado deberá transformase en actor para tratar de representar algún problema o situación actual que le interese, como pueden ser el acoso escolar o el cambio climático. El tema será elegido por el alumnado, pero el tutor o tutora puede también proponer temas. Así, en la primera sesión, deberán elegir entre todas las personas participantes un tema a tratar y, para ello, realizarán una lluvia de ideas. Los temas pueden ser muy variados y, por tanto, el personal docente deberá orientar a sus estudiantes. (30 minutos)

Posteriormente, se repartirán los papeles con la clase dividida en dos. En una parte estarán estudiantes defendiendo una postura, y en la otra, estudiantes defendiendo la contraria. Cada grupo deberá inventarse un pequeño teatro, donde participen la totalidad de sus integrantes, No debe durar más de 15 minutos. Para ensayar, dispondrán de la mitad de esta primera clase y la clase de tutoría siguiente (1 hora y media). Por último, en la última sesión se interpretarán ambos teatros (media hora). La clase finaliza con un debate a mano alzada, donde cada estudiante expondrá su punto de vista sobre el tema que se ha tratado. (30 minutos) 
doi: http://doi.org/10.15359/ree.24-1.12

URL: http://www.una.ac.cr/educare

CORREO: educare@una.cr

\section{Evaluación}

En cuanto a la evaluación de esta propuesta didáctica, se deberá valorar, por una parte, el ejercicio, tarea y actuación del alumnado; y por otra, la idoneidad y adecuación de la propuesta didáctica y el trabajo del profesorado al cargo de la tutoría.

En el caso de la evaluación del alumnado, se valorarán las actitudes y la disposición mostrada durante la realización de las actividades. Para ello, el profesor o profesora utilizará básicamente la herramienta de la observación sistemática. Así, recogerá, en su diario de clase, la forma de trabajar del alumnado, su trabajo individual y grupal, su participación en clase y la disposición mostrada, la motivación, el respeto a las demás personas y el uso del turno de la palabra, la autonomía de actuación, la correcta expresión oral y escrita, la asistencia a la excursión y otros aspectos. En resumen, a modo de evaluación continua, en el diario se recogerá todo lo relacionado a cómo se resuelve el alumnado en clase.

Asimismo, se propone que el personal docente también realice una evaluación final en el diario. Mediante esta, se valorará lo trabajado durante el curso, para detectar el grado de aprendizaje adquirido respecto a la cultura participativa. Igualmente, y en relación con los objetivos fijados inicialmente, se pueden destacar los siguientes criterios de evaluación planteados en forma de sencilla rúbrica (ver Tabla 4).

Tabla 4: Rúbrica de evaluación

Criterios de evaluación

\section{Registro de cumplimiento}

Sí No A veces

Observaciones

1. Participa de forma activa en las actividades de clase.

2. Conoce el deber y el derecho de la participación individual y grupal.

3. Realiza actividades utilizando TIC de forma correcta y autónoma.

4. Realiza una presentación y representación colectiva.

5. Interactúa en grupo mediante el diálogo.

6. Respeta las opiniones de los demás compañeros y compañeras de clase.

7. Afianza su autonomía personal.

Nota: Elaboración propia. 
Finalmente, para evaluar la idoneidad y adecuación de la propuesta, parece conveniente que el maestro o maestra registre también si las iniciativas planteadas se han llevado a cabo. Pues si se elaboran unas reglas de convivencia, pero no se cumplen; o si se diseña una excursión, pero finalmente no se hace, todo parece indicar que la participación es ficticia. En este caso, el personal docente deberá realizar una reflexión profunda para superar las deficiencias en futuras experiencias de impulso de la participación en el aula.

\section{Consideraciones finales}

Conviene señalar, en primer lugar, que la propuesta, que ha sido puesta en marcha hasta el momento de manera parcial, es flexible y puede ser modificada por el personal docente para adaptarla al grupo-clase con el que trabaje durante el curso escolar. Realizando los cambios necesarios en función del estadio madurativo del grupo, la secuencia de actividades puede ser utilizada con alumnado de otros cursos de educación primaria o, incluso, del primer ciclo de la educación secundaria. Y es que, para potenciar la cultura y las competencias participativas de estudiantes, trabajar proyectos de este tipo durante todos los cursos académicos, realizando las adaptaciones necesarias, parece lo más adecuado. La propuesta pretende servir, por lo tanto, de punto de partida para el personal docente interesado en esta cuestión.

Asimismo, como se ha expuesto, participar supone tomar parte en las decisiones colectivas que afectan la vida de los miembros de una comunidad. Solo participando activamente en nuestro contexto conseguiremos construir sociedades más justas y democráticas. Ahora bien, este esfuerzo común no surge de la nada, sino que requiere un sustrato que se genera en los agentes socializadores básicos, con especial relevancia de la escuela.

La participación es un derecho reconocido a la niñez por diferentes instrumentos legales, lo que demuestra su importancia. Y existen instrumentos para medirla. No hay excusa, por tanto, para no trabajar esta cuestión en las aulas, más allá de acciones aisladas, como la elección del delegado o la delegada de clase, de un modo reflexivo, continuado y sistemático. Así, la propuesta que se plantea pretende contribuir a esa visión de la escuela como "comunidad participativa"(Puig, 1996) para lo cual proporciona una secuencia clara y ordenada de actividades que potencien las competencias participativas del alumnado durante todo un curso escolar.

Siguiendo estudios como los de Freinet (1972) o Freire (1997), se impone potenciar la cultura democrática y participativa desde la escuela, si queremos vivir en sociedades verdaderamente democráticas y participativas. Y para llegar a esta utopía, cada vez más cercana, se requieren propuestas concretas y ordenadas, que reivindiquen el espacio de la participación como contenido escolar. 
doi: http://doi.org/10.15359/ree.24-1.12

URL: http://www.una.ac.cr/educare

CORREO: educare@una.cr

\section{Referencias}

Arnstein, S. R. (1969). A ladder of citizen participation. Journal of the American Planning Association, 35(4), 216-224. doi: https://doi.org/10.1080/01944366908977225

Blas, A. e Ibarra, P. (2006). La participación: Estado de la cuestión. Cuadernos Hegoa, 39, 1-44. Recuperado de https://www.ehu.eus/ojs/index.php/hegoa/article/view/10754/10046

Blondiaux, L. (2008). Le nouvel esprit de la démocratie. Actualité de la démocratie participative. Paris: Le Seuil.

Brady, H. (1999). Political participation. En J. P. Robinson, P. R. Shaver y L., S. Writgtsman (Eds.), Measures of political attitudes (pp. 737-801). San Diego: Academic Press.

Castilla, V. (2016). Alumnado con voz y voto. Cuadernos de pedagogía, 467, 34-36.

Coiduras, J. L., Balsells, M.À., Alsinet, C. y Urrea, A. (2016). La participación del alumnado en la vida del centro: Una aproximación desde la comunidad educativa. Revista Complutense de Educación, 27(2), 437-456. doi: https://doi.org/10.5209/rev RCED.2016.v27.n2.46353

Cortés, J. (2001). Construcción socio-histórica y conceptualización jurídica de la infancia. En Corporación opción, infancia y derechos humanos: Discurso, realidad y perspectiva (pp. 6170). Santiago de Chile: Corporación Opción.

Cunill, N. (1991). La participación ciudadana. Caracas: CLADE.

Dale, R. A. (1999). Participación infanto-juvenil: Un reto social. OPS/OMS.

de la Riva, F. y Moreno, A. (2018). Una experiencia de educación para la participación. Revista Communiars, 1, 43-51. Recuperado de https://drive.google.com/file/ d/19vVNnHAqYnNH5X9FdmY4-BiBpyT4VYER/view

Etxebarría-Kortabarría, I. (2015). Las claves para una escuela participativa. Una propueta para $6^{\circ}$ de educación primaria (Trabajo de grado). Universidad Internacional de La Rioja, Logroño. Manuscrito inédito.

Freinet, C. (1972). Por una escuela del pueblo. Barcelona: Fontanella.

Freire, P. (1997). Política y educación. Madrid: Siglo XXI.

Gaitán, Á. (1998): Protagonismo infantil. En B. Abegglen y R. Benes (Comps.), La participación de niños y adolescentes en el contexto de la Convención sobre los derechos del niño: Visiones y perspectivas (pp. 85-104). Florencia, Italia: UNICEF. Recuperado de https://www.unicef-irc. org/publications/pdf/bogota.pdf 
García, M. y Prado, B. (2013). El alumnado tiene la palabra. Cuadernos de Pedagogía, 432, 33-35.

Gyarmati, G. (1992). Reflexiones teóricas y metodológicas en torno a la participación. Estudios sociales, 73(3), 9-28.

Hart, R. A. (1993). La participación de los niños. De la participación simbólica a la participación auténtica (Serie Ensayos Innocenti, 4). Recuperado de https://www.unicef-irc.org/ publications/538-la-participaci\%C3\%B3n-de-los-ni\%C3\%B1os-de-la-participaci\%C3\%B3nsimbolica-a-la-participaci\%C3\%B3n.html

Hart, R. A. (1997). Children's participation: The theory and practice of involving young citizens in community development and environmental care. Londres: Unicef.

Hart, R. A. (2001). La participación de los niños en el desarrollo sostenible. Barcelona: Pau Educación.

Jiménez, M. y García, P. (2015). Los efectos de los procesos participativos en la sociedad civil. La opinión de los expertos. Córdoba: Instituto de Estudios Sociales Avanzados.

Ley Orgánica 1/1990, de 3 de octubre, de Ordenación General del Sistema Educativo. BOE, núm. 238, de 4 de octubre de 1990, 24172, pp 28927- 28942. Recuperado de https://www.boe. es/boe/dias/1990/10/04/pdfs/A28927-28942.pdf

Ley Orgánica 2/2006, de 3 de mayo, de Educación (LOE). BOE, núm. 106, de 4 de mayo de 2006, 7899, pp. 1-110. Recuperado de https://www.boe.es/buscar/pdf/2006/BOE-A-2006-7899consolidado.pdf

Ley Orgánica 8/2013, de 9 de diciembre, para la mejora de la calidad educativa (LOMCE) . BOE, núm. 295, de 10 de diciembre de 201, 12886, pp. 1-64. Recuperado de https://www.boe. es/buscar/pdf/2013/BOE-A-2013-12886-consolidado.pdf

Ministerio de Educación, Política Social y Depote, Secretaría de Estado de Política Social, Familias y Atención a la Dependencia y a la Discapacidad e IMSERSO. (2008). La participación social de las personas mayores. Madrid: IMSERSO. Recuperado de www.imserso.es/InterPresent2/ groups/imserso/documents/.../11005partsocialmay.pdf

Morán, R. (2018). (Coord.). Participación y exclusión política: Causas, mecanismos y consecuencias. Tirant lo Blanch.

Naciones Unidas. (2006). Convención sobre los derechos del niño. Madrid: UNICEF Comité español. Recuperado de https://www.un.org/es/events/childrenday/pdf/derechos.pdf

Novella, A. M. (2012). La participación infantil: Concepto dimensional en pro de la autonomía ciudadana. Teoría de la Educación. Educación y Cultura en la Sociedad de la Información, 13(2), 380-403 Recuperado de http://revistas.usal.es/index.php/eks/article/view/9015/9259 
doi: http://doi.org/10.15359/ree.24-1.12

URL: http://www.una.ac.cr/educare

CORREO: educare@una.cr

Pérez, A. y Figueroa, H. (2005). Construcción de participación infantil en niñas y niños del Hogar de Cristo, Antofagasta (Tesis de Licenciatura). Universidad Católica del Norte, Chile.

Puig, J. M. (1996). La escuela, comunidad participativa. Cuadernos de Pedagogía, 253, 28-34.

Real Academia Española (RAE). (2001). Diccionario de la lengua española (23a ed.). Recuperado de http://www.rae.es/

Tenjo, J., Ribero, R. y Sanabria, P. (1998). Participación, desempleo y mercados laborales en Colombia. Bogotá: Archivos de macroeconomía, 81.

Torcal, M., Montero J. R. y Teorell, J. (2006). La participación política en España: Modos y niveles en perspectiva comparada. Revista de estudios políticos, 132, 7-41. Recuperado de https:// recyt.fecyt.es/index.php/RevEsPol/article/view/45507/27026

Trilla, J. y Novella, A. (2001). Educación y participación social de la infancia. Revista lberoamericana de Educación, 26, 137-166. Recuperado de https://rieoei.org/historico/documentos/rie26f. htm

Verba, S. y Nie, N. (1972). Participation in America: Political democracy and social equality. Nueva York: Harper \& Row. 